\title{
Performance of jet reconstruction and calibration in first ATLAS data at a centre-of-mass energy of $7 \mathrm{TeV}$
}

\section{S. Eckweiler* on behalf of the ATLAS Collaboration}

Institut für Physik, Universität Mainz, Mainz

E-mail: Sebastian.Eckweiler@cern.ch

\begin{abstract}
We report on first results of jet reconstruction and jet calibration in ATLAS in proton-proton collisions produced at a centre-of-mass energy of $7 \mathrm{TeV}$ at the LHC. Jets are reconstructed with the anti-kt jet algorithm and need to satisfy a few selection criteria to reject backgrounds. We compare the data in detail to Monte Carlo simulations and estimate uncertainties on the jet energy scale and jet energy resolution. In addition, in-situ techniques are used to assess the energy scale and resolution.
\end{abstract}

35th International Conference of High Energy Physics

July 22-28, 2010

Paris, France

* Speaker. 


\section{Jets in ATLAS}

Jets are reconstructed using the Anti-kT algorithm [1] with a distance parameter $\mathrm{R}=0.6$. They undergo a very efficient chain of quality cuts to identify jets heavily affected by e.g. noise bursts [2]. In early data the calibration scheme employed by ATLAS consists of a simple $\left(p_{T}, \eta\right)$-dependent calibration, referred to as $E M+J E S$, which is derived using Monte Carlo (MC) simulations. Figure 1 shows the $p_{T}$ spectrum corresponding to an integrated luminosity of about $400 \mu \mathrm{b}^{-1}$.

For future data analysis ATLAS is studying more sophisticated calibration schemes than the simple $\left(p_{T}, \eta\right)$-dependent scheme. Those schemes make use of more detailed information about a given jet and thus provide a significant improvement of the jet energy resolution. They are described in detail in [2]. One of these, the GCW+JES scheme, attempts to correct for the noncompensating nature of the calorimeters by weighting each jet constituent cell according to their energy density. To be able to use such calibration schemes, one has to be confident that the exploited features such as the energy density in the calorimeter are well described by the MC simulation. To evaluate the modeling of the properties used for a given scheme, the jet energy before and after calibration is compared in data and MC. Figure 1 shows that these quantities agree to a very good degree of $2 \%$ for the $G C W+J E S$ method.

\section{Jet Energy Resolution and Scale Uncertainty}

The amount of data provided by the LHC is not yet sufficient for fully data-driven jet calibration methods such as Photon/Z + Jet balance. The uncertainty on the jet energy scale is therefore evaluated by studying a variety of MC predictions [3], each describing distinct features of the physics and detector simulation differently from the nominal MC simulation. The total uncertainty on the jet energy scale amounts to about $6 \%$ for $p_{T}^{\text {jet }}>100 \mathrm{GeV}$, as shown in Fig. 2. The dominant contributions in this momentum range arise from the uncertainty related to the hadronic shower model and the absolute scale of the calorimeters, which was evaluated in test-beam measurements.

The jet energy resolution can be measured in data using clean dijet events [4], selected by requiring two leading jets in the event, together with a third jet with $p_{T, 3}<p_{T, 3}^{\text {cut }}$. To determine the resolution the width $\sigma_{A}$ of the $p_{T}$-asymmetry $A$ of the two leading jets in the event, defined as $A\left(p_{T, 1}, p_{T, 2}\right)=\frac{p_{T, 1}-p_{T, 2}}{p_{T, 1}+p_{T, 2}}$, is measured. Here $p_{T, 1}$ and $p_{T, 2}$ are the randomly ordered, transverse momenta of the two jets. It can be shown that the resolution as a function of $\bar{p}_{T}=\frac{1}{2}\left(p_{T, 1}+p_{T, 2}\right)$ amounts to $\sqrt{2} \sigma_{A}$. The complete measurement is repeated for values of $p_{T, 3}^{\text {cut }}$ between $7 \mathrm{GeV}$ and $20 \mathrm{GeV}$ and the observed resolution in each $\bar{p}_{T}$-bin is extrapolated to $p_{T, 3}^{\text {cut }} \rightarrow 0$. The result is shown in Fig. 2 and compared to MC simulation: the overall agreement is at a level of $14 \%$, where the measurement for $\bar{p}_{T}>40 \mathrm{GeV}$ is presently statistically limited. A second measurement presented in this figure is described in detail in [4]. Observed differences in the resolutions measured with both methods arise from different sensitivities of the methods to the imbalance on the particle-jet level. 

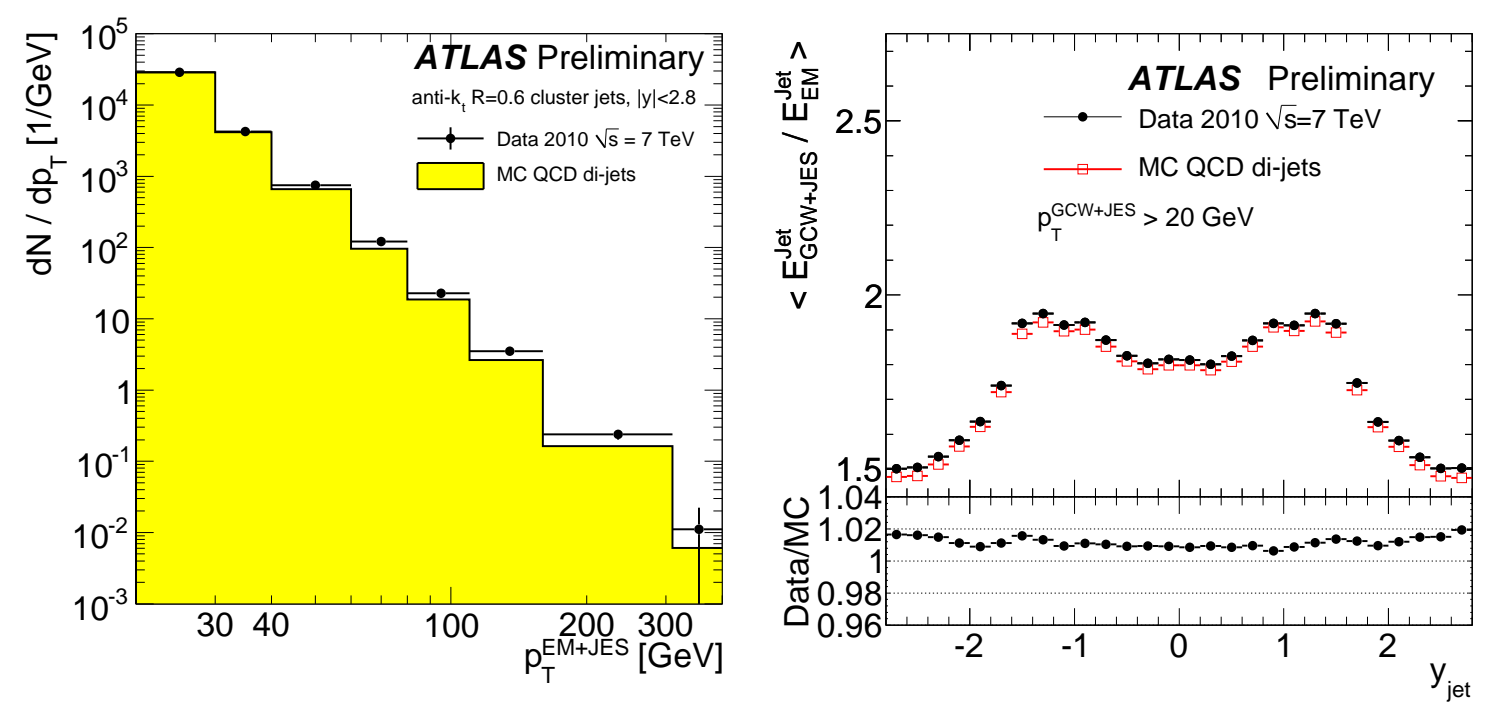

Figure 1: Jet $p_{T}$ distribution using the $E M+J E S$ calibration scheme (left) and the ratio of jet energies before and after the application of the GCW+JES calibration scheme (right).
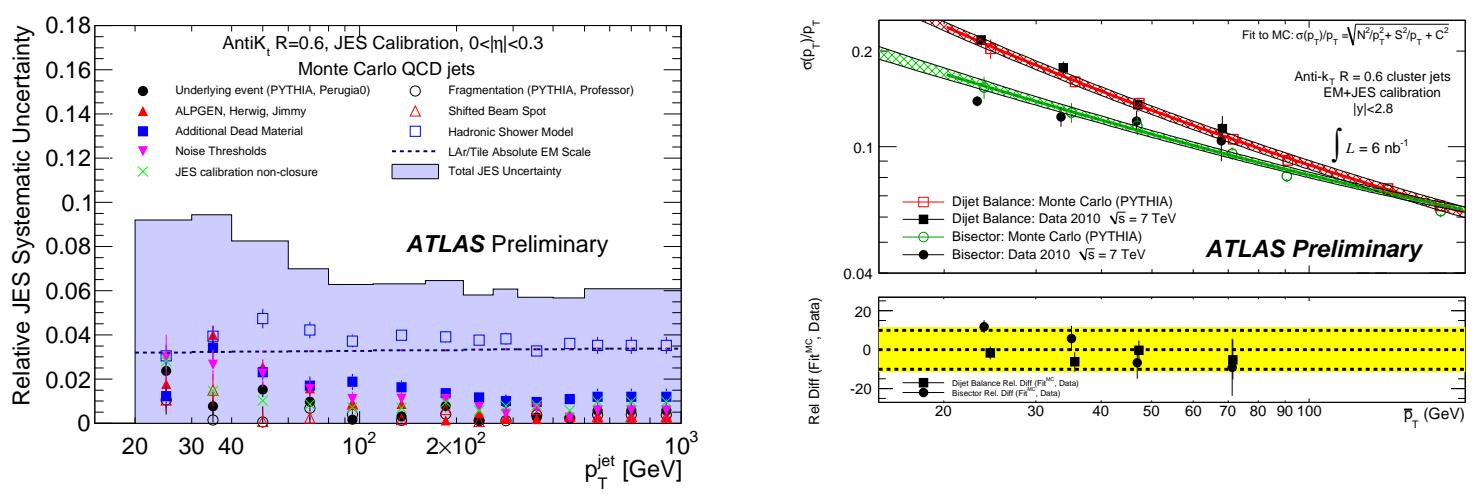

Figure 2: Total relative jet energy scale uncertainty as a function of jet $p_{T}$ and its contributions (left) and measured jet energy resolution compared to Monte Carlo simulations (right).

\section{References}

[1] M. Cacciari, G. P. Salam, G. Soyez, The anti- $k_{\mathrm{T}}$ jet clustering algorithm, JHEP 0804 (2008) 063

[2] The ATLAS Collaboration, Properties of Jets and Inputs to Jet Reconstruction and Calibration with the ATLAS Detector Using Proton-Proton Collisions at $\sqrt{s}=7 \mathrm{TeV}$, ATLAS-CONF-2010-053

[3] The ATLAS Collaboration, Jet energy scale and its systematic uncertainty for jets produced in proton-proton collisions at $\sqrt{s}=7 \mathrm{TeV}$ and measured with the ATLAS detector, ATLAS-CONF-2010-056

[4] The ATLAS Collaboration, Jet energy resolution and selection efficiency relative to track jets from in-situ techniques with the ATLAS Detector Using Proton-Proton Collisions at a Center of Mass Energy $\sqrt{s}=7 \mathrm{TeV}$, ATLAS-CONF-2010-054 